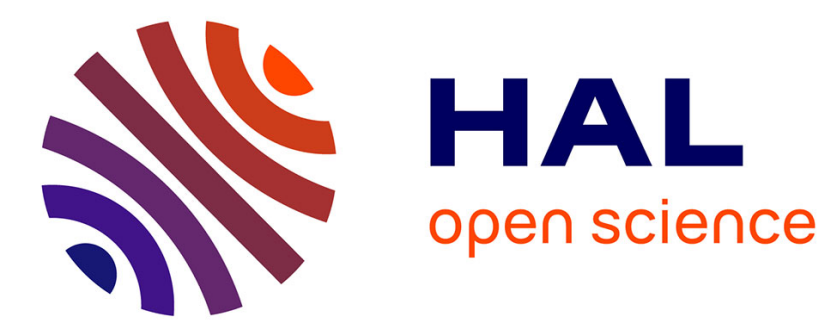

\title{
Histoire et mémoire de la guerre civile dans l'historiographie espagnole contemporaine
}

\author{
Mercedes Yusta Rodrigo
}

\section{To cite this version:}

Mercedes Yusta Rodrigo. Histoire et mémoire de la guerre civile dans l'historiographie espagnole contemporaine. Matériaux pour l'histoire de notre temps, 2003, Espagne: la mémoire retrouvée (1975-2002), 70, pp.51-58. 10.3406/mat.2003.402452 . hal-00918845

HAL Id: hal-00918845

https://hal-univ-paris8.archives-ouvertes.fr/hal-00918845

Submitted on 15 Dec 2013

HAL is a multi-disciplinary open access archive for the deposit and dissemination of scientific research documents, whether they are published or not. The documents may come from teaching and research institutions in France or abroad, or from public or private research centers.
L'archive ouverte pluridisciplinaire HAL, est destinée au dépôt et à la diffusion de documents scientifiques de niveau recherche, publiés ou non, émanant des établissements d'enseignement et de recherche français ou étrangers, des laboratoires publics ou privés.

\section{(ㅇ)(1) $\$$}

Distributed under a Creative Commons Attribution - NonCommercial - NoDerivatives 44.0 


\title{
Persée
}

http://www.persee.fr

\section{Histoire et mémoire de la Guerre civile dans l'historiographie espagnole contemporaine}

\author{
Mercedes Yusta Rodrigo \\ Yusta Rodrigo Mercedes. Histoire et mémoire de la Guerre civile dans l'historiographie espagnole contemporaine. In: \\ Matériaux pour l'histoire de notre temps. 2003, N. 70. Espagne : la mémoire retrouvée (1975-2002). pp. 51-58.
}

Voir l'article en ligne

L'historiographie de la guerre civile espagnole s'est construite en opposition à la politique de la mémoire de la dictature franquiste. Au fil des années et des confrontations avec l'histoire « officielle », cette historiographie a évolué vers des problématiques de plus en plus complexes, où les questions liées à la violence politique et à la gestion de la mémoire l'em portent sur les études plus événementielles. Néanmoins, cette évolution ne s'est pas produite sans heurts entre quelques « anciens combattants » du camp des vainqueurs et leurs héritiers, d'une part, et d'autre part ceux qui veulent écrire une histoire renouvelée, qui viserait à récupérer la mémoire et la dignité des vaincus. L'article montre cette évolution, ainsi que ses rapports à la politique et à la mémoire collective.

\section{Avertissement}

L'éditeur du site «PERSEE » - le Ministère de la jeunesse, de l'éducation nationale et de la recherche, Direction de l'enseignement supérieur, Sous-direction des bibliothèques et de la documentation - détient la propriété intellectuelle et les droits d'exploitation. A ce titre il est titulaire des droits d'auteur et du droit sui generis du producteur de bases de données sur ce site conformément à la loi n $98-536$ du 1 er juillet 1998 relative aux bases de données.

Les oeuvres reproduites sur le site «PERSEE » sont protégées par les dispositions générales du Code de la propriété intellectuelle.

Droits et devoirs des utilisateurs

Pour un usage strictement privé, la simple reproduction du contenu de ce site est libre.

Pour un usage scientifique ou pédagogique, à des fins de recherches, d'enseignement ou de communication excluant toute exploitation commerciale, la reproduction et la communication au public du contenu de ce site sont autorisées, sous réserve que celles-ci servent d'illustration, ne soient pas substantielles et ne soient pas expressément limitées (plans ou photographies). La mention Le Ministère de la jeunesse, de l'éducation nationale et de la recherche, Direction de l'enseignement supérieur, Sous-direction des bibliothèques et de la documentation sur chaque reproduction tirée du site est obligatoire ainsi que le nom de la revue et- lorsqu'ils sont indiqués - le nom de l'auteur et la référence du document reproduit.

Toute autre reproduction ou communication au public, intégrale ou substantielle du contenu de ce site, par quelque procédé que ce soit, de l'éditeur original de l'oeuvre, de l'auteur et de ses ayants droit.

La reproduction et l'exploitation des photographies et des plans, y compris à des fins commerciales, doivent être autorisés par l'éditeur du site, Le Ministère de la jeunesse, de l'éducation nationale et de la recherche, Direction de l'enseignement supérieur, Sous-direction des bibliothèques et de la documentation (voir http://www.sup.adc.education.fr/bib/). La source et les crédits devront toujours être mentionnés. 


\section{Histoire et mémoire de la Guerre civile}

\section{Mercedes YUSTA RODRICO \\ dans l'historiographic espagnole contemporaine}

\section{$\mathbf{U}_{\text {ne }}$ "guerre de mots $^{1}$ "}

En 1995, vingt ans après la mort de Franco, une émission de la chaîne privée espagnole Telecinco réunissait l'historien Javier Tusell, spécialiste de la période franquiste? ${ }^{2}$ la députée de Esquerra Republicana de Catalunya Pilar Rahola, Joaquín Arango, intellectuel progressiste et alors directeur du Centre de recherches sociologiques (CIS), et trois des plus acharnés apologistes du régime franquiste Angel Palomino, Fernando Vizcaino Casas $^{3}$ et le président de la Fundación nacional Francisco Franco, Joaquín Gutiérrez Cano - , pour participer à un débat sur la dictature animé par le journaliste Fernando Jáuregui. La question de départ, aussi incroyable que cela puisse paraître, était la suivante: "Avec Franco, vivions-nous mieux? ", phrase qui, sous sa forme affirmative, était devenue un lieu commun des nostalgiques de la dictature. Le débat tourna très vite à l'affrontement personnel, ce qui était prévisible vu les positions absolument irréconciliables des uns et des autres. Le déclenchement des hostilités se produisit à propos des origines de la Guerre civile et, plus significativement, à propos des morts de part et d'autre, que les invités se jetaient à la figure.

Il y a plusieurs enseignements à tirer de l'analyse de cette émission qui témoigne d'une situation très particulière de l'Espagne par rapport à sa propre mémoire historique. D'abord, l'absurdité du sujet proposé pour lancer le débat révèle un certain manque de consensus par rapport à la mémoire de la dictature ; si ce consensus existait, la proposition ne serait même pas concevable. Nous pouvons tirer la même conclusion du fait qu'une chaîne de diffusion nationale ait laissé un espace de parole à des gens qui, dans un pays comme la France, par exemple, seraient immédiatement accusés de négationnisme. Ensuite, les propos tenus par les défenseurs du régime pendant l'émission (et dans leurs publications) ont été démentis depuis très longtemps par une historiographie académique et compétente. Javier Tusell, représentant lui-même de cette historiographie professionnelle, et peu suspect de " gauchis-

MERCEDES YUSTA RODRICO est docteur en histoire et en études hispaniques. Chercheur à I'Université de Paris VIII et enseignante à l'Institut d'études politiques de Paris. Spécialiste des mouvements antifranquistes des années 1940. Auteur des livres Guerrilla y resistencia campesina. La resistencia armada contra el régimen de Franco en Aragón, 1939-1952 (2003) et La guerra de los vencidos. El maquis en el Maestrazgo furolense, 1940-1950 (1999). me ", insistait particulièrement sur ce point, sans réussir à se faire écouter au milieu du tohu-bohu qu'était devenu le plateau après une heure d'émission. Et, finalement, on constate que la Guerre civile continue d'être le sujet de controverse récurrent : qui l'a commencée, quelles furent ses causes, qui a fait le plus de morts et d'atrocités, en un mot, à qui la faute?

Mais surtout, l'intérêt de l'anecdote est qu'elle témoigne, après vingt ans de démocratie, d'une césure entre l'activité des historiens espagnols et ce qui est proposé au public à travers les médias ; entre une historiographie solide, qui est arrivée à des conclusions non contestées de l'intérieur de la profession, et la mémoire collective des Espagnols, où les mythes mis en place par le franquisme sur la Guerre civile, sur Franco ou sur le régime lui-même peuvent trouver à un moment donné un certain écho. Encore aujourd'hui, des historiens issus de la dictature et formés par elle continuent à publier des ouvrages où ils réécrivent une version de la Guerre civile et du franquisme conforme à la mythologie du régime et se réapproprient une histoire dont ils considèrent avoir été dépossédés ${ }^{4}$. Certes, ils ne font pas partie du débat historique " sérieux " : ils ne sont ni cités, ni pris en compte par les historiens professionnels. II n'en reste pas moins que cette production existe, que ces livres se vendent et que leurs auteurs trouvent un écho dans une partie de la presse et des médias qu'il serait imprudent de négliger. Et cela a certainement des conséquences qui, de temps en temps, se reflètent dans la production historiographique. À différentes reprises, des historiens spécialistes du franquisme et de la Guerre civile se sont sentis obligés de publier des travaux dont l'objectif avoué et explicite était de contester ces mythes et d'en démontrer l'absence de fondement ${ }^{5}$.

Néanmoins, force est de constater aussi l'incroyable progrès accompli par l'historiographie académique depuis la mort de Franco en partant d'une situation de notable infériorité. Elle a dû surmonter les difficultés liées à l'accès aux sources, " séquestrées " par les autorités, la bureaucratie et les héritiers du régime " séquestre " qui, dans certains cas, continue, comme c'est le cas pour la Fundación nacional Francisco Franco, citée plus haut, qui a le monopole des papiers aussi bien personnels qu'officiels du général. Elle a dû aussi s'imposer face au discours officiel, fort de quarante ans de monopole de l'histoire, et surtout aux

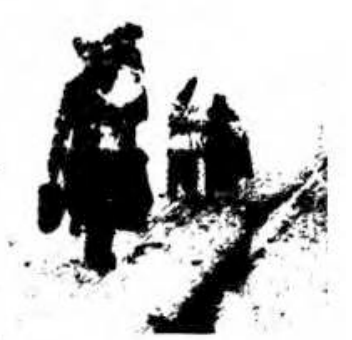

Partie de la couverture du catalogue de l'exposition Exilio, qui eut lieu à Madrid du 17 septembre aul 28 octobre 2002. Fondation Pablo Iglesias, Musée national centre d'art reina Sofia.

En raison de leur importance, les notes ont été mises à la fin de l'article. 
conséquences mêmes de la Guerre civile. Car la guerre et surtout la dictature de Franco ont entrainé - à part la répression, le manque de libertés, l'impossibilité d'écrire une histoire qui dévierait d'un iota de la version officielle - la rupture avec la tradition historiographique libérale, c'est-à-dire un trou béant qui engloutit tout l'héritage de l'école rationaliste, de I'Institución libre de Enseñanza, de Rafael Altamira ${ }^{6}$. L'historiographie espagnole sur le XXe siècle allait passer à la trappe jusqu'aux années 1960, exception faite des œuvres écrites à la gloire du régime franquiste et l'histoire de la Guerre civile dut se construire sur ce néant. De plus, elle se vit dès ses débuts confrontée à l'appareil de propagande franquiste qui réagit avec violence à la perte de son monopole dans la gestion de la mémoire.

\section{Historiens anglo-saxons et exilés espagnols}

Couverture de la version française de A Concise History of the Spanish Civil War, publiée en anglais en 1973. Ruedo Ibérico a publié également en 1974 des éditions en castillan et en catalan.
Jusqu'au début des années 1960, I'histoire de la guerre civile espagnole avait été écrite, presque exclusivement, par les vainqueurs de cette guerre et ses héritiers. On y retrouve des auteurs comme Luis Bolín ou Joaquín de Arrarás. Le premier, organisateur du voyage de Franco vers la Péninsule en 1936 à bord de l'avion Dragon rapide et attaché de presse du QG du général Franco pendant la Guerre civile, se spécialisa dans I'occultation des atrocités commises par l'armée dite " nationale ", comme les massacres de Badajoz ou le bombardement de Guernica. Son œuvre España : los años vitales, publiée tardivement (en 1967), reprenait les arguments utilisés par la propagande franquiste, et donc par lui-même, pendant la guerre. Quant à Arrarás, il codirigea une œuvre monumentale sur la Guerre civile (huit volumes) rédigée pendant les années qui suivirent le conflit et intitulée, de façon significative, Historia de la Cruzada Española, et par la suite il écrivit une histoire de la Seconde République, rééditée plusieurs fois (dont la dernière en 1970), qui la présentait comme une période d'anarchie et justifiait ainsi le coup d'État de 1936'.

Cette historiographie franquiste était construite autour de deux axes. D'une part, le caractère inévitable du conflit, lequel, selon la version franquiste, aurait été déclenché pour épargner à l'Espagne la révolution soviétique qui se préparait dans les cuisines de la République et pour préserver l'unité nationale. D'autre part, son caractère de " croisade ", parfaitement assumé par l'Église espagnole, qui avait prêté tout son appareil de propagande aux insurgés pendant le conflit et donnait toujours légitimité et appui au régime du général Franco - bien que certaines voix dissidentes se fassent bientôt entendre. C'est précisément ce caractère de " croisade " accordé à la Guerre civile qui va être la cible d'un des premiers travaux sur le conflit fait en dehors de l'influence du régime et qui va provoquer un tournant dans l'historiographie " officielle ". II s'agit de l'œuvre de l'historien nord-américain Herbert R. Southworth, El Mito de la cruzada de Franco, publiée en 1963 à Paris par un éditeur espagnol en exil, José Martínez, alma mater des mythiques éditions Ruedo Ibérico ${ }^{8}$.

Ruedo Ibérico avait amorcé sa trajectoire éditoriale deux ans auparavant avec deux titres consacrés aussi à la Guerre civile, et écrits dans une optique bien différente de celle des historiens officiels du régime. II s'agissait de La Guerra Civil española, de Hugh Thomas, et El Laberinto español, de Gerald Brenan. Avec cet ouvrage, originellement publié en anglais en 1943, Brenan fut le premier à analyser la Guerre civile comme un affrontement à caractère social, directement lié aux problèmes du monde rural espagnol et susceptible d'être replacé dans le long terme'. Quant à l'ouvrage de Thomas, il allait devenir " la Bible de la gauche espagnole ${ }^{10}$ » ; des dizaines d'exemplaires (comme ce fut le cas par la suite de tous les livres publiés par Ruedo Ibérico) commencèrent à traverser la frontière clandestinement et allèrent fournir les arrière-boutiques des librairies engagées des principales villes espagnoles. Mais ce fut l'ouvrage de Southworth qui provoqua de façon concrète la réaction du régime de Franco. Dans son livre, Southworth dénonçait la propagande utilisée par la droite espagnole pendant le conflit, en particulier l'instrumentalisation faite par les « nationaux » qui consistait à présenter la Guerre civile comme une guerre de défense des valeurs de la religion, menacées par les hordes athées et marxistes. En réponse, et face à la menace que supposait un tel ouvrage pour l'un des mythes fondateurs du régime franquiste, celui-ci créa tout un département, sous la tutelle du jeune et dynamique ministre de I'Information et du Tourisme, Manuel Fraga Iribarne, dont le seul but était de contrecarrer les affirmations de Southworth. À la tête de ce département fut placé le journaliste Ricardo de la Cierva, qui, confronté à " une bataille perdue avec Southworth, a écrit jusqu'à présent quatre-vingts livres en défense du régime de Franco ". On peut néanmoins considérer que cet événement contribua à un certain renouvellement de I'historiographie franquiste, qui s'adonna à un effort de modernisation du discours, certes très limité mais qui présentait comme nouveauté radicale de proposer un partage des responsabilités de la Guerre civile sur le modèle " on a tous été coupables" " .

Ruedo Ibérico continua de publier des ouvrages sur la Guerre civile, dont d'autres titres de Southworth et des ouvrages d'auteurs tels que Gabriel Jackson, lan Gibson, Stanley Payne ou Burnet Bollotten ${ }^{12}$. Ce dernier auteur apporta à l'étude de la Guerre civile la dimension révolutionnaire présente dans le camp républicain, en analysant en particulier le rôle joué par le Parti communiste d'Espagne dans l'étouffement de cette révolution manquée, un aspect qui avait déjà été abordé dans l'étude pionnière de Pierre Broué et Émile Temime $^{13}$. Quant à Gabriel Jackson, il avait publié en Angleterre, en 1965, une large étude qui embrassait la période républicaine et la Guerre civile et où la presse républicaine avait été utilisée comme source historique pratiquement pour la première fois. Cela faisait de son 
livre un travail de recherche très important et original, à la différence du livre de Thomas qui avait surtout un caractère vulgarisateur.

En tout cas, cette prolifération d'ouvrages sonnait définitivement le glas de l'exclusivité franquiste dans la construction du récit historique de la Guerre civile et, surtout, constituait une "démolition méthodique des mensonges sur lesquels les franquistes avaient bâti leur histoire $^{14}$ ". Dorénavant, la brèche ouverte allait s'agrandir de plus en plus. Ce renouveau allait prendre un élan définitif grâce à un autre exilé, Manuel Tuñón de Lara, professeur à l'université de Pau. Dans son séminaire annuel, qui débuta en 1970, les jeunes (et moins jeunes) historiens espagnols non conformistes finirent l'apprentissage qu'ils avaient entrepris avec la lecture des ouvrages publiés par José Martínez.

\section{Le renouveau historiographique sous la Transition}

Or, la production sur la guerre de cette génération éduquée sous la tutelle de Manuel Tuñón de Lara mais aussi de José María Jover et Miguel Artola, qui prônèrent quant à eux le renouvellement de l'historiographie espagnole à partir de l'histoire sociale - allait attendre jusqu'au début des années 1980 pour voir le jour, les colloques de Pau portant essentiellement sur I'histoire espagnole antérieure à 1936. Au moment de la mort de Franco et du déclenchement du processus de démocratisation, c'étaient surtout les œuvres des auteurs anglo-américains qui étaient à portée de main. Aux premiers, Thomas, Brenan, Jackson, mais aussi Raymond Carr, qui publia en 1977 son œuvre The Spanish Tragedy, avait succédé une deuxième génération, celle de Edward Malefakis, Paul Preston, Frances Lannon ou Ronald Fraser. Ce dernier publia en 1979 un livre qui allait ouvrir l'historiographie de la Guerre civile vers d'autres perspectives, jusque-là inexplorées. II s'agit de Blood of Spain. An Oral History of The Spanish Civil War, publié la même année en Espagne avec un titre tiré d'un poème de Luis Cernuda qui exprimait à la perfection l'état d'esprit qui allait caractériser la Transition à la démocratie par rapport à la mémoire de la Guerre civile : Recuérdalo tú y recuérdalo a otros ${ }^{15}$

Le livre de Fraser est en effet exemplaire du renouveau historiographique et aussi de l'orientation qu'allait prendre cette historiographie novatrice qui voyait le jour après la mort du dictateur. Cet ouvrage est une " histoire orale de la Guerre civile ", d'où son originalité : Fraser sillonne l'Espagne en cherchant des témoignages directs de personnes ayant vécu la guerre d'un côté et de l'autre; son livre constitue donc un recueil de récits liés à la mémoire de la Guerre civile, une mémoire nettement populaire aussi bien des vainqueurs que des vaincus. En quelque sorte, cet ouvrage est l'aboutissement d'un processus amorcé avec les livres de Thomas ou Jackson, lequel consistait à pré- senter un bilan " égalitaire " du conflit. Sans acquitter les militaires conspirateurs et, en particulier, Franco, auxquels revient en dernière instance la responsabilité d'avoir déclenché le conflit en se soulevant contre la légitimité républicaine, ces ouvrages présentent un "partage égalitaire des responsabilités ${ }^{16}$ " : on constate que les deux parties ont commis des crimes, on retrouve l'horreur des deux côtés et en même temps, en regardant le conflit de plus près, à travers la voix de ceux qui luttèrent en première ligne, on découvre que chacun avait ses raisons, que tous croyaient lutter pour un idéal juste. Comme dans I'historiographie franquiste menée par Ricardo de la Cierva (mais avec des méthodes et des arguments bien différents), ici aussi on découvre que tout le monde était coupable (ce qui revient aussi à dire que personne ne l'était).

Tout cela reflète assez bien l'ambiance qui présida à la Transition : partage des responsabilités et " pacte d'oubli » (qui, comme le signale Santos Juliá, ne signifiait nullement oublier la Guerre civile ou arrêter d'en écrire l'histoire, mais une sorte d' " amnistie " de part et d'autre sur les faits commis pendant la dictature ${ }^{17}$ ). Pacte d'oubli certes discutable, car on n'oublie que ce qui n'est pas utile - les crimes du franquisme, le droit de cité des combattants clandestins de la guérilla tandis que la guerre et ses horreurs, susceptibles d'une utilisation politique, on ne les oublie certainement pas $^{18}$. Dans l'édition espagnole de Fraser, cette injonction au souvenir, à la fois individuel et partagé ( recuérdalo tú, recuérdalo a otros ) qui s'exprimait en empruntant la voix d'un poète mort en exil, indique cette primauté de la mémoire. Néanmoins, l'utilisation de la mémoire faite par l'historiographie est quand même loin du fantôme de la Guerre civile exhibé comme une menace pointant à I'horizon par les secteurs réformistes du régime qui pilotent alors la transition à la démocratie. Selon Julio Aróstegui, "plus que la mémoire active de la guerre civile de 1936-1939 [...], ce fut un type de discours - et non un type de mémoire - qui fut réellement opératoire ou important pendant la transition. Ce discours faisait de la Guerre civile comme événement type la seule alternative à l'intelligibilité de la "Transition " comme " réforme" des institutions existantes. La mémoire de la Guerre civile a été dans une large mesure un stratagème au service des attentes des secteurs réformistes et de leur discours $^{19}$ ". En même temps, il est vrai que ce discours va profiter du bilan égalitaire établi au début par l'historiographie, ainsi que du fait que ces œuvres présentaient en quelque sorte une "connexion non vérifiée entre l'échec de la République et son inévitable résolution en Guerre civile 20 , conclusion très profitable au service d'un processus de transition qui allait dérober aux citoyens toute sorte de débat sur la nature de l'État et donc sur l'alternative monarchie/république.

En outre, il faut tenir compte du fait que cette historiographie s'est construite au travers d'une lutte dialectique contre la propagande franquiste, que la mort

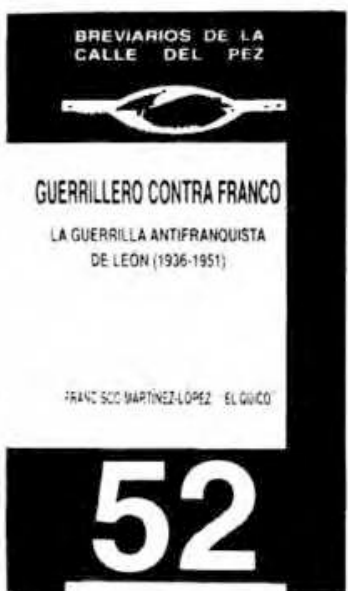

Édition espagnole de 2002 du livre de Francisco MartinezLópez, Guerillero contre Franco, publié en français en 2000 aux Éditions Syllepse. 
de Franco ne fit nullement taire, bien au contraire : quelques-unes des œuvres les plus militantes datent de la Transition ou des années qui la suivirent. Cela eut pour conséquence, entre autres, cette tendance de I'historiographie professionnelle à "équilibrer la balance $n$ de peur de tomber à son tour dans des excès opposés. Néanmoins, la balance historiographique va bientôt s'incliner pour des causes multiples.

D'une part, à la fin des années 1970 commencent à paraître les monographies et les thèses doctorales des historiens espagnols formés sous l'égide de Tuñón de Lara, de Jover et d'Artola ou de Raymond Carr. Même si ceux qui s'occupent de la Guerre civile ne sont pas les plus nombreux, tous portent un regard nouveau sur l'histoire récente de l'Espagne et contribuent à miner encore plus un héritage franquiste déjà très mal en point. Et notamment les débats sur la nature du régime franquiste (qui, d'ailleurs, reviennent aujourd'hui à la mode) vont provoquer une avalanche de travaux. Le débat s'amorce autour d'un article de Juan José Linz, * Una teoría de régimen autoritario. El caso de España ", paru en 1978 et publié originairement en anglais en 1964. L'auteur qualifiait le régime de Franco $\mathrm{d}^{\prime}$ " autoritaire " et le plaçait ainsi dans le groupe de régimes susceptibles d'un regard favorable des Alliés pendant la guerre froide, le soustrayant ainsi à la dénomination de "fasciste ", ce qui signifiait une réinterprétation générale du régime lui-même autant que de la nature de la Guerre civile dans le contexte européen $^{21}$. La critique à cette terminologie se développa notamment pendant les années 1970 et signifiait un effort épistémologique d'envergure - mené par des personnalités telles que Juan Martínez Alier, Juan José Carreras ou Manuel Tuñón de Lara lui-même -, qui allait modifier profondément la production historiographique et qui continuera jusqu'à nos jours. Les études sur la guerre se placeront aussi sous l'influence de ces efforts de théorisation, et désormais le conflit ne sera plus expliqué comme un événement conjoncturel mais comme le fruit d'un déséquilibre structurel entre développement et retard, comme une guerre de classes ou comme une crise de domination des élites ${ }^{22}$.

Par ailleurs, la " guerre de mots " entre les historiens se transforme en " guerre de chiffres * quand, audelà des études plus ou moins générales sur le déroulement de la guerre, les historiens, et d'abord ceux favorables au régime, se donnent pour tâche de comptabiliser les victimes des deux camps, en vue de confirmer ou de démentir le chiffre devenu mythique du " million de morts » mais surtout pour dégager le caractère plus ou moins meurtrier de chacune des factions (pratique vite désignée en milieu historiographique sous l'appellation quelque peu ironique de " compter les morts "). Le premier à emprunter cette voie est Ramón Salas Larrazábal, historien militaire et ancien combattant sous les drapeaux de Franco pendant la guerre. Son étude, Pérdidas de guerra, est publiée en 1977 et fait partie de cette tactique de l'his- toriographie franquiste qui vise à placer les conséquences de la guerre sous la responsabilité partagée des deux parties ${ }^{23}$. Mais la méthodologie de Salas Larrazábal fut vite contestée par les historiens non franquistes : les sources utilisées, celles de l'Institut national de statistique (franquiste) privilégiaient les morts " nationaux ", et tous les morts n'ont pas été inscrits dans les registres à un moment ou à un autre, comme le pensait Salas Larrazábal. Au contraire, des études ultérieures ont montré que, très souvent, les morts de la répression franquiste dans les zones occupées pendant la guerre tantôt n'étaient pas inscrits (on sait maintenant combien les fosses communes ont été nombreuses dans les zones sous contrôle franquiste), tantôt avaient été enregistrés dans une rubrique qui alléguait une autre cause de mort, telle que : " asphyxie ", "fracture du crâne "... Au début des années 1990, des historiens de la répression franquiste ont démontré que souvent ce genre d'inscriptions correspondait aux morts victimes de la répression franquiste ${ }^{24}$. Ce qui ne veut pas dire que ces pratiques (notamment les fosses communes) n'aient pas existé aussi du côté républicain. La différence est que la victoire franquiste entraîna l'ouverture des fosses pour " enterrer chrétiennement " les martyrs de la cause nationale, et en même temps une gigantesque recherche sur tous les crimes et exactions commises du côté républicain, en vue d'établir le dossier d'un énorme procès contre tous les responsables, la Causa General, et de ce fait la répression républicaine est assez bien connue. Les traces de la violence franquiste sont, au contraire, bien plus difficiles à retrouver après quarante ans de silence.

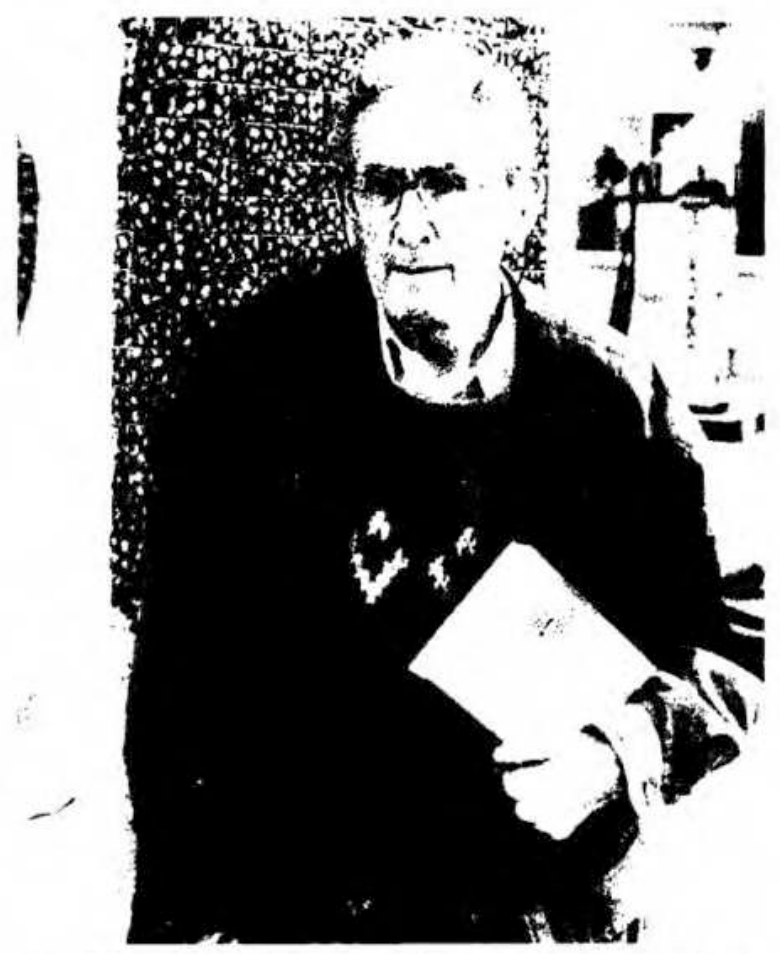

Eduardo Pons Prades avec son dernier livere Las guerras de los niños republicanos, photo José Maria Azkárraga, extrait de son livre Perder la guerra, Valence, Ediciones del dia de la foto, 1999 (ㄷ) José Azkárraga), 
En conclusion, les chiffres de Salas Larrazábal établirent que les morts franquistes étaient plus nombreux, et placèrent ainsi la répression républicaine pendant la guerre au-dessus de celle pratiquée dans la zone franquiste. Ce qui, de surcroît, justifiait la violence franquiste comme une riposte aux exactions commises en zone républicaine, notamment contre le clergé. À l'époque, ce fut Alberto Reig Tapia qui se chargea de contester ces chiffres et surtout la méthodologie de ce travail dans un article publié dans la revue Sistema ${ }^{25}$. Mais le chemin était désormais ouvert et plusieurs jeunes historiens $s^{\prime} y$ engouffrèrent, entreprenant un travail minutieux et de portée locale, unique façon d'aboutir au rétablissement de chiffres susceptibles d'approcher la réalité en croisant des sources multiples. Ces travaux sur la répression allaient constituer un véritable chantier de renovation de l'étude de la Guerre civile, et cette fois-ci les historiens espagnols allaient prendre le devant.

\section{Les années 1980 et 1990 : entre l'explosion de l'histoire locale et l'histoire commémorative}

Les années comprises entre 1981 et 1996 ont connu une véritable explosion de travaux concernant la Guerre civile, des travaux adoptant une multiplicité d'optiques mais pour la plupart limités à un cadre local. La route de cette historiographie est désormais jalonnée par les commémorations : le cinquantième anniversaire de la proclamation de la République en 1981 et celui de l'éclatement de la Guerre civile en 1986 provoquent une avalanche de travaux de circonstance et, la décennie suivante, le soixantième anniversaire des deux éphémérides redouble le phénomène. Cette production, qui va vite atteindre un volume très important, se caractérise donc par le choix d'un cadre réduit et aussi par un empirisme de plus en plus marqué, s'inscrivant dans la continuité de la " guerre de chiffres " déclenchée par Salas Larrazábal. Par contre, le débat théorique se fait de plus en plus rare, remplacé par des propositions méthodologiques concernant, surtout, l'étude de la répression, qui a donc été, de façon assez paradoxale, un agent de renouvellement de l'historiographie tout en paralysant en quelque sorte le débat épistémologique. Selon Julián Casanova, " en réalité, ce fut l'empirisme qui s'imposa ${ }^{26}$ ".

À l'occasion du cinquantenaire se produisent deux événements historiographiques d'une importance majeure : d'une part, un colloque à Salamanque organisé par la Société d'études de la Guerre civile et du franquisme (SEGUEF), sous le titre significatif de " Histoire et mémoire de la guerre civile d'Espagne " dont les actes sont publiés deux ans plus tard; et, d'autre part, la parution du livre La Guerra Civil española. 50 años después, dirigé par Manuel Tuñón de Lara, I'un des pères spirituels de la rénovation de l'historiographie de la guerre civile espagnole (et de l'histo- riographie espagnole tout court) ${ }^{27}$. Mais, mis à part ces ouvrages et la synthèse effectuée par Pierre Vilar dans la collection "Que sais-je ?", adressée à un public français ${ }^{28}$, il $n^{\prime} y$ a pas de grande œuvre de synthèse qui voie le jour à cette occasion-là (d'autres œuvres parues ayant un caractère vulgarisateur, comme celles publiées par El País et Historia 16). Le dynamisme du débat théorique cède la place à la question de la caractérisation ou de la « nature " du régime franquiste. Qui plus est, le climat politique ne semble pas favorable à une discussion publique sur la Guerre civile, susceptible de mettre en question les théories, encore assez répandues, de la " responsabilité partagée ". Et cela malgré la présence d'un gouvernement socialiste, qui, de façon assez surprenante, publie dans El País, à l'occasion du cinquantenaire un communiqué faisant écho à cette idée de la faute partagée et du respect des idéaux des combattants des deux côtés, lesquels pensaient tous entreprendre une lutte pour la liberté et la justice. L'heure n'est pas à la mise en question de cette façon de voir le conflit.

Il est vrai, pourtant, que pendant les années 1980 se produit un renouvellement de I'historiographie avec, notamment, l'émergence de nouveaux sujets du récit historique, tels que les paysans ou les femmes : les collectivisations anarchistes en Aragon font l'objet de la thèse de doctorat de Julián Casanova, tandis que Marie Nash entreprend l'étude du groupe de femmes anarchistes Mujeres libres avant même la mort de Franco, tout comme Giuliana di Febo sur la résistance des femmes face à la dictature ${ }^{29}$. La résistance au franquisme (pendant ou après la Guerre civile) devient aussi objet d'étude et on voit apparaître les premières recherches sur le phénomène méconnu de la guérilla ${ }^{30}$. Tous ces travaux incluent des groupes sociaux traditionnellement considérés comme minoritaires, incorporent les sources orales et se nourrissent des analyses proposées par les courants historiographiques les plus novateurs, tels que la New Left Review ou les History Workshops. Les sources orales sont aussi une source privilégiée dans les travaux sur la répression, surtout ceux qui se développent dans les marges de I'histoire académique, comme le travail réalisé par le collectif Altaffaiya Kultur Taldea, exemplaire d'un souci qui date de ce moment historique de construction (ou reconstruction) régionale : le souci de certaines communautés d'écrire leur propre histoire ${ }^{31}$. La mise en place de l'Estado de las Autonomías ne sera pas étrangère à ce développement de l'histoire locale, qu'elle va même favoriser par une politique d'attribution de bourses ou de subventions à la recherche. Chaque communauté va revendiquer sa propre mémoire historique (ce qui ne va pas aller sans confrontation avec le pouvoir central) et la Guerre civile, déchirement fondateur et enjeu de significations politiques et sociales, va être au centre de ces nouvelles politiques de la mémoire. Et dans certains cas, par exemple dans les communautés historiques dont la forte personnalité culturelle a été réprimée sous le franquisme, comme la Catalogne ou le

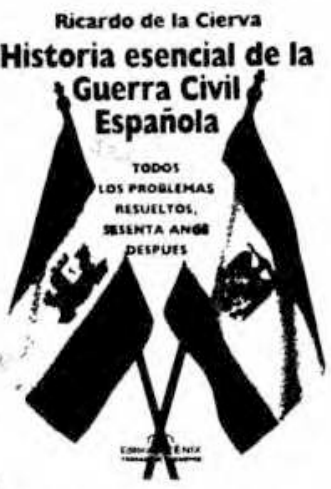

Ricardo de la Cierva Histoire essentielle de la guerre civile espagnole. Tous les problèmes résolus, soixante ans après. 
lieux de mémoire dont le franquisme avait marqué l'Espagne ? II est évident que le Valle de los Caídos n'est pas le Panthéon. Et, de manière plus générale, les particularités de la construction nationale espagnole se prêtent mal à ce genre de discours historique, comme Carlos Serrano l'a parfaitement démontré 40 .

Néanmoins, suivre les traces de cette histoire inscrite dans l'espace aussi bien que dans le temps n'a rien d'une tâche inutile : cela permet, par exemple, de révéler à quel point les vainqueurs de la Guerre civile s'adonnèrent à un travail de " destruction-reconstruction ", selon la terminologie de Josefina Cuesta, où, une fois de plus, la mémoire était au centre des enjeux. La création des "lieux de mémoire " franquistes revenait à effacer la mémoire dès vaincus et à la substituer par une mémoire mythique, créée ad hoc et qui visait d'une part à justifier le soulèvement de 1936 et de l'autre à l'inscrire dans la lignée des gestes héroïques de l'Espagne impériale. En fait, il faudrait parler plutôt d'un processus de " destruction-réappropiation ", puisque le franquisme ne construit rien mais il pille ici et là des éléments idéologiques, des lambeaux d'histoire et des symboles qui, de ce fait, deviendront problématiques une fois la démocratie instaurée. Les récents problèmes concernant l'énorme drapeau espagnol placé par ordre du ministre de la Défense à la Plaza de Colón, à Madrid, et la polémique dont cette mise en place a été entourée, n'en sont qu'un exemple ${ }^{41}$.

Cela a un rapport direct avec la problématique de la gestion de la mémoire et I' « usage public de l'histoire " (selon la terminologie de Habermas), laquelle, après I'intérêt soulevé en Allemagne et en Italie, intéresse de plus en plus la communauté historienne espagnole et s'est imposée comme un élément incontournable de tout congrès, séminaire ou rencontre scientifique : le débat sur les usages publics, ou politiques, de I'histoire, ainsi que sur les institutions auxquelles reviendrait le droit de gérer et administrer la mémoire des citoyens, fait rage. Au centre de la polémique se trouve la réappropiation de l'histoire par les différents nationalismes péninsulaires (y compris le nationalisme espagnol, ou " espagnoliste »), qui était en fait la toile de fond du fameux débat sur "l'enseignement des sciences humaines " qui eut lieu en $1999^{42}$. Et on se demande si, au-delà des problèmes qui relèvent de la construction de l'État-nation espagnol par les libéraux au XIX e siècle et de l'institutionnalisation parallèle de la discipline historique, il n'y aurait pas une certaine gêne liée à l'usage fait par le franquisme de quelques idées et symboles (y compris l'idée même de l' " unité nationale ") qui, sans lui, auraient pu être perçus comme constituants d'une identité collective espagnole. Une fois de plus, on observe à travers ces débats comment la Guerre civile et ses conséquences ont inscrit des ruptures durables dans notre perception de l'histoire.

En ce qui concerne l'historiographie la plus récente, la polémique est ouverte entre ceux qui parlent d'un " pacte de silence "sur lequel se serait construit le consensus démocratique et ceux qui soutiennent qu'il ne s'agit nullement de silence, mais d'un oubli nécessaire pour construire une cohabitation pacifique après la disparition du dictateur. En fait, si on regarde le volume de la production qui concerne la Guerre civile (plus de 3575 références de monographies, actes de colloques et articles de revue entre 1975 et 1995) on peut difficilement parler de "silence " d'une manière générale. En tout cas, avec les débats sur la nature du franquisme (qui reviennent à/dans l'actualité historiographique), les études sur la violence politique, les polémiques sur les indemnisations ou l'ouverture des fosses, nous sommes probablement plus près que jamais de la situation qui eut lieu en Allemagne au moment de l'historikerstreit. Le fait que le Congrès des députés ait récemment (novembre 2002) approuvé une résolution qui exprimait la reconnaissance morale envers les victimes de la Guerre civile et de la répression franquiste, et qui approuvait des aides économiques pour les exilés et les « niños de la guerra », est le symbole du long chemin parcouru entre les premières études qui s'attaquèrent à I'histoire "officielle " et le rétablissement de la dignité des vaincus.

En même temps, il est vrai qu'il y a un certain décalage entre les avancées expérimentées par l'historiographie et ce qu'on pourrait appeler la * mémoire publique " de la Guerre civile, une mémoire qui embrasserait la mémoire collective (multiple, hétérogène, où les mythes trouvent une large place) et la mémoire dite " officielle " (homogène, univoque, véhiculée à travers les actes politiques et les déclarations d'intentions). Et ce décalage alimente des polémiques qui vont bien audelà de ce qui serait le travail de l'historien pour prendre la voie de la revendication. D'une part, les versions révisionnistes de la guerre d'Espagne trouvent une place et une crédibilité dans cet espace flou ; d'autre part, les collectifs des vaincus et surtout ceux qui ont été doublement vaincus, comme les anarchistes ou les membres du POUM, ont l'impression que leur mémoire à eux n'a pas trouvé de place dans le discours historien sur la Guerre civile. Dans ce dernier cas, il y a une sorte de confusion qui s'installe entre histoire et mémoire, et en même temps cela devrait interpeller I'historien sur les matériaux qu'il utilise pour construire le récit historique - dont, bien souvent, la mémoire des survivants - et l'usage qu'il en fait ${ }^{\text {t3 }}$.

En tout cas, malgré les efforts qui ont été faits et I'indéniable actualité que revêtent toujours ces débats, on peut constater une certaine ignorance du public en général, et surtout des plus jeunes, par rapport à cette mémoire et à cette histoire. Pour revenir au débat de la chaîne espagnole Telecinco par lequel nous avons engagé cette réflexion, l'émission s'ouvrait sur une enquête auprès des étudiants universitaires de la Complutense, où certains d'entre eux avouaient que, pour eux, Franco était un personnage aussi lointain et méconnu que Ponce Pilate. Mais cela renvoie à un problème qui relève de l'enseignement de l'histoire et de la gestion de la mémoire et non plus de I'historiographie elle-même.

\section{Le labyrinthe espagnol}

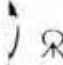

Gerald Brenan, Le Labyrinthe espagnol. Origines sociales et politiques de la Guerre civile, Paris, Ruedo Ibérico, 1962, couverture. 
1. Expression empruntẻe à Paul Preston, = Guerra de palabras : los historiadores ante la guerra civil española s, in Paul Preston (ed.), Revolución y guerra en España, 1931-1939, Madrid, Alianza Editorial, 1986, pp. 15-24.

2. Javier Tusell étant un auteur très prolifique, nous ne citerons ici que quelques-uns de ses ouvrages les plus significatifs: La Oposición democrática al franquismo. Barcelone, Planeta, 1977 ; Franco y los católicos: la política interior española entre 1945-1957. Madrid, Alianza Editorial, 1984 ; Javier Tusell et Genoveva Queipo de Llano, Franco y Mussolini : la politica española durante la segunda guerra mundial. Barcelone, Planeta, 1985; La Dictadura de Franco, Madrid, Alianza Editorial, 1988 (réédité plusieurs fois); Franco, España y la ll guerra mundial : entre el eje y la neutralidad. Madrid, Temas de hov, 1995, ou sa récente biographie d'Alphonse XIII, en collaboration avec Genoveva Queipo de Llano, Alfonso XIII : el rey polémico. Madrid, Taurus, 2001.

3. Le premier est l'auteur notamment de Caudillo, Barcelone, Planeta, 1992 et Defensa del Alcázar. Una epopeya de nuestro tiempo. Barcelone, Planeta, 1995. Le deuxième, très prolifique, est l'auteur, entre autres, de De * camisa vieja * a chaqueta nueva : crónica de una evolución ideológica, Barcelone, Planeta, 1976; 1975, el año en que franco murió en la cama, Barcelone, Planeta, 1992 ou ... Y al tercer año resucitó. Novela-ficción, Barcelone, Planeta, 1995.

4. Comme exemple, seulement deux titres, très récents, đu plus prolifique de ces auteurs, Ricardo de la Cierva, qui témoignent parfaitement de l'esprit qui anime ses écrits: El 18 de julio no fue un golpe militar fascista : no existia la legalidad republicana. Deformación y violación sistemática de la memoria histórica de los Españoles: todas las pruebas, Tolède, Fénix, 2000, et Historia esencial de la guerra civil espaniola : todos los problemas resueltos, sesenta años después, Tolède, Fénix, 1996, ouvrage qui suscita une réaction exaspérée de l'historien Julio Aróslegui dans son article * La guerra de don Ricardo y otras guerras n, Hispania, LVII/2, $n^{\circ} 196$, 1997, pp. 777-787, où il le qualifiait de c publiciste inépuisable et épuisant s. 5. Ainsi le fit, très tôt, Herbert R. Southworh avec El Mito de la cruzada de Franco, Paris, Ruedo thérico, 1963, ouvrage réédité en Espagne en 1986 par Plaza \& Janés et dont nous allons parler plus loin. Plus récemment, voir Alberto Reig Tapia, Memoria de la Guerra Civil. Los mitos de la tribu, Madrid, Alianza Editorial, 1999, ou il revient sur les grands clichés de la mythologie franquiste de la Guerre civile, tels que la menace de la révolution communiste ou la défense hẻroïque de l'Alcazar de Tolède. 6. Voir Gonzalo Pasamar, Historiografia e ideologia en la posguerra española : La ruptura de la tradición libera, Saragosse, Prensas Universitarias de Zaragoza, 1991.

7. Luis Bolín, España, los años vitales, Madrid, Espasa-Calpe, 1967 ; Joaquin de Arrarás (dir.littéraire), Ciriaco Pérez Bustamante, Carlos Saenz de Tejada (dir. artistique) Historia de la Cruzada Española. Madrid, Españolas, 1939-1944 (8 vol.); ; Joaquin de Arrarás, Historia de la Segunda República Española, Madrid, Editora Nacional, 1956 |rééd. en 1968 (deux fois) et en 1970|.

8. Herbert R. Southworth, El Mito de la Cruzada de Franco, op. cit.. (Éd. française, Le Mythe de la croisade de Franco, Paris, Ruedo lbérico, 1964.)

9. Hugh Thomas, La Guerra Civil española, Paris, Ruedo Ibérico, 1961 ; Gerald Brenan El Laberinto español : Antecedentes políticos y sociales de la guerra civil, París, Ruedo thérico, 1962

10. Paul Preston, "Guerra de palabras... *, op. cit, p. 17

11. Paul Preston, \& El lavado de cerebro de Francisco Franco s, El Pais, 21 novembre 1999. Sur ce renouveau de I'historiographie franquiste, voir Paloma Aguilar Fernández, Memoria y olvido de la guerra civil española, Madrid, Alianza Editorial, 1996, pp. 108-112.

12. Herbert R., Southworth, Antifalange. Estudio crítico de a Falange en la guerra de España s, de M. Garcia Venero, Paris, Ruedo lbérico, 1967, et la Destrucción de Guernica : periodismo, propaganda, diplomacia e historia, Paris, Ruedo lbérico, 1977 Ian Gibson, La Represión nacionalista de Granada en 1936 y la muerte de Federico Garcia Lorca, Paris, Ruedo lbérico, 1971 ; Gabriel Jackson, Breve Historia de la guerra civil de España, Paris, Ruedo lbérico, 1974 ; Stanley Payne, Falange. Historia del fascismo español, Paris, Ruedo lbérico, 1965, et Los Militares y la política en la España contemporánea, Paris, Ruedo lbérico, 1968; Bumet Bollotten, La Révolution espagnole, I: La Gauche et la lutte pour le pouvoir, Paris, Ruedo lbérico, 1977.

13. Pierre Broué, Émile Temime, La Révolution et la Guerre d'Espagne, Paris, Ėditions de Minuit, 1961. (trad, esp.: La Revolución y la Guerra de España, Mexico, FCE, 1962.)

14. Paul Preston, "Guerra de palabras... s, op. cit., p. 17

15. Ronald Fraser, Blood of Spain. An Oral History of The Spanish Civil War, Londres, 1979 ; Recuérdalo tú y recuérdalo a otros. Historia oral de la Guerra civil española. Barceione, Crítica, 1979

16. Idée développée par Julián Casanova dans son article * Guerra civil, ¿lucha de clases? El difícil ejercicio de reconstruir el pasado s, Historia Social, $n^{\circ} 20$, automne 1994, pp. 135-150.

17. Voir Santos Juliả, s Postiranquisme ou société démocratique $\%$, Vingtième siècle. Revue d'histoire, $\mathrm{n}^{\circ} 74$, avril-juin 2002, pp. 5-12.

18. Comme ce sujet va ètre repris dans d'autres articles de cette revue, je vais juste signaler les éléments en rapport avec le discours de l'historiographie.

19. Julio Aróstegui, * La mémoire de la guerre civile et du franquisme dans l'Espagne démocratique " Vingtième siècle. Revue d'histoire, $n^{\circ} 74$, avril-juin 2002 , pp. $31-42$, p. 39.

20. Julián Casanova, * Guerra civil... s, op. cit., p. 137, n. 3.

21. Juan José Linz, * Una teoria de rêgimen autoritario. El caso de Esparì *. in Stanley G. Payne, Politica y sociedad en la España del siglo XX, Madrid, Akal, 1978 pp. 205-263. Paru originairement dans E. Allardt et Y. Littunen (eds.), Cleavages, ideologies and Party Systems. Contribution to Comparative Political Sociology, Helsinki, 1964
22. Voir le débal sur la nature du régime franquiste in Angel Cenarro, Cruzados y camisas azules. Los origenes del franquismo en Aragón, 1936-1945, Saragosse, Prensas Universitarias de Zaragoza, 1997, pp. 16-25. Le renouveau théorique des travaux sur la guerre dans Julián Casanova, * Guerra civil... 3, op. cit.

23. Ramón Salas Larrazábal, Pérdidas de guera, Barcelone, Planeta, 1977. Voir ce débat dans Angela Cenarro, "Muerte y subordinación en la España franquista, El imperio de la violencia como base del eNuevo Estado *, Historia Social, n 30, 1998, pp. 5-22.

24. Je pense notamment aux travaux de Juliản Casanova et son équipe, de Josep Maria Solé i Sabaté ou de Francisco Moreno.

25. Alberto Reig Tapia, * Consideraciones metodológicas para el estudio de la represión franquista en la guerra civil $2,5 i s t e m a, n^{\circ} 33$, novembre 1979. 11 a repris ses arguments dans quelques ouvrages postérieurs, comme Ideología e historia. (Sobre la represión franquista y la guerra civil, Madrid, Akal, 1984, ou Violencia y terror. Estudios sobre la guerra civil española, Madrid, Akal, 1990.

26. Juliain Casanova, × Guerra civil... 1, op. cit., p. 150.

27. Julio Aróstegui (coord.), Historia y memoria de la guerra civil. Encuentro en Castilla y León, Valladolid, Junta de Castilla y Leỏn, 1988; Julio Aróstegui, Ángel Viñas, Gabriel Cardona y Josep M. Bricall, La Guerra Civil española 50 años después, Barcelone, Labor, 1985.

28. Pierre Vilar, La Guerre civile espagnole, Paris, PUF, " Que sais-je ? s, 1984. 29. Julián Casanova, Anarquismo y revolución en la sociedad rural aragonesa, 1936-1938, Madrid, Siglo XXI, 1985; Mary Nash, * Mujeres libres *, España 1936-1939, Barcelone, Tusquets, 1976 ; Giluliana di Febo: Resistencia y movimiento de mujeres en España 1936-1976, Barceione, Icaria, 1979.

30. Pourtant, le premier travail s académique * est dû à un Allemand, Hartmut Heine, A guerrilla antifranquista en Galici, Vigo, Xerais, 1980.

31. Altaffaiya Kultur Taldea, Navarra, 1936. De la esperanza al terror, Pampelune, Altaffaiya Kultur Taidea, 1986, 2 vol.

32. Alberto Reig Tapia, Memoria de la guerra civil..., op. cit., pp. 323-324. 33. Enrique Moradiellos, La Perfidia de Albión. El gobiema británico y la Guerra civil española, Madrid, Siglo XXI, 1996.

34. Julio Aróstegui, * Mémoire de la guerre civile... s, op. cit., p. 41.

35. Julián Casanova (ed.), Morit, matar, sobrevivir, La violencia en la dictadura de Franco, Madrid, Temas de Hoy, 2002, p. 50.

36. Santos Julia (coord.), Victimas de la Guerra civil, Madrid, Temas de Hoy, 1999; Secundino Serrano, Maquis. Historia de la guerrilla antifranquista. Madrid, Ternas de Hoy, 2001.

37. Javier Ugarte, La Nueva Covadonga insurgente. Origenes sociales y culturales de la sublevación de 1936 en Navarra y el Pris Vasco, Madrid, Biblioteca Nueva, 1998 ; Michael Richards, Un tiempo de silencio. La Cuerra civil y la cultura de la represión en la Esparia de Franco, Barcelone, Crítica, 1999.

38. Une des premières études est celle de Clicerio Sánchez Recio, Justicia y Guerra en España : Los tribunales populares (1936-1939), Alicante, Diputación de Alicante, Instituto de Cultura Juan Gil Albert, 1991. Voir aussi José Luis Ledesma, = El lasire de un pasado incautado: (ab)uso politico, memoria e historiografia de la represión republicana zi in Preactas VI Congreso de la Asociación de Historia Contemporánea : Usos públicos de la Historia, Saragosse, Prensas Universitarias de Zaragoza, 2002, pp. 140-155.

39. Paloma Aguilar Fernández, Memoria y olvido..., op. cit.

40. Carlos Serrano, El Nacimiento de Carmen. Simbolos, mitos y nación, Madrid. Taurus, 1999.

41. Josefina Cuesta, * La destrucción de la memoria de la ll República (1936-1944) D, in Marie-Claude Chapul et Thomas Gomez (dir.), Histoire et mémoire de la Seconde République espagnole, Nanterre, Presses universitaires de Paris X, 2002, pp. 347-374 ; Esther Casanova, - La conformación politica en los espacios públicos durante la inmediata posguerra , in Preactas VI Congreso de la Asociación de Historia Contemporánea : Usos públicos de la Historia, Saragosse, Prensas Universitarias de Zaragoza, 2002, pp. 74-84. Le débat autour de la mise en place de l'énorme drapeau espagnol $\left(300 \mathrm{~m}^{2}\right.$ sur un mât de $50 \mathrm{~m}$ de hauteur) eut lieu au début du mois d'octobre 2002 ; voir léditorial du joumal El País du 4 octobre 2002, où le journal qualifie la mise en place de ce drapeau de s plouc s et fait un rapprochement entre cet usage du drapeau et l'usage fait par le régime de Franco.

42. La bibliographie générée par ce débat est tellement ample que nous ne citerons que quelques ouvrages à titre d'exemple. On trouve un bon état de la question, publié dans le vif de la polémique, dans Juan Sisinio Pérez Garzón et al., La Gestión de la memoria. La historia de España al servicio del poder, Barcelone, Crítica, 2000. Voir aussi Carlos Forcadell (ed.), Nacionalismo e Historia. Saragosse, Institución Fernando el Católico, 1998; Carlos Fortadell, Ignacio Peiró (eds)., Lecturas de la historia. Nueve reflexiones sobre historia de la historiografía, Saragosse, institución Fernando el Católico, 2002, ou l'article de Pedro Ruiz, * Les usages politiques de l'histoire en Espagne. Formes, limites et contradictions n, in François Hartog et jacques Revel (dir.), Les Usages politiques du passé, Paris, Éditions de l'EHESS, 2001, pp. 129-156. Le dernier congrès de l'Association d'histoire contemporaine, dont les actes seront prochainement publiés, a eu aussi pour sujet * Les usages publics de l'Histoire :.

43. Voir Javier Rodrigo, "En el limbo de la historia. La memoria de los campos de concentración franquistas, entre el olvido oficial y el uso público s, in Preactas VI Congreso de la Asociación de Historia Contemporánea: Usos públicos de la Historia. Saragosse, Prensas Universitarias de Zaragoza, 2002, pp. 235-251. 